\title{
Molecular detection of hemotropic mycoplasmas (hemoplasmas) in domestic cats (Felis catus) in Romania
}

Mirela Imre ${ }^{1 \dagger}$, Cristina Văduva ${ }^{2 \dagger}$, Gheorghe Dărăbuș ${ }^{1}$, Sorin Morariu' ${ }^{1}$, Viorel Hermann ${ }^{3}$, Judit Plutzer ${ }^{4}$, Tijana Suici $^{1}$, Philippa J P Lait ${ }^{5}$ and Kálmán Imre 6* $^{*}$

\begin{abstract}
Background: The hemotropic mycoplasmas (hemoplasmas) of the genus Mycoplasma are recognized as important bacteria that parasitize red blood cells, causing hemolytic anemia in many mammalian species, including cats. No information is available concerning the presence of feline hemoplasma infections in cats in Romania. Thus, the objective of the present study was to provide data on the occurrence and molecular characterization of hemotropic mycoplasmas in client-owned cats in Romania.
\end{abstract}

Methods: Blood samples from 51 unhealthy cats, originating from Timişoara Municipality, Romania, were screened for the presence of hemoplasmas using conventional polymerase chain reaction (PCR) targeting the 16S rRNA gene and sequencing assays. PCR-positive samples were subsequently analyzed by phylogenetic and population genetic analysis.

Results: Molecular analysis revealed 11 (21.6\%) positive samples, consisting of 8 (72.7\%) Candidatus Mycoplasma haemominutum and 3 (27.3\%) Mycoplasma haemofelis confirmed positives. Candidatus Mycoplasma turicensis was not detected, and no co-infections were registered. No significant associations $(p>0.05)$ were found between the hemoplasma infection status and age, gender, breed, presence of ectoparasites, feline leukemia virus/feline immunodeficiency virus positivity of cats, or the sampling season. However, outdoor access was positively associated $(p=0.049)$ with infection and could be considered a risk factor $(\mathrm{OR}=4.1)$ in acquiring feline hemotropic mycoplasmas. Phylogenetic analysis revealed that our sequences clustered with those selected from the GenBank database in two distinct clades. The registered population genetic indices were strongly supportive of the great variance in sequences between the recorded Mycoplasma species.

Conclusions: The findings support the occurrence of feline hemoplasma infections in previously uninvestigated territories of Europe, providing useful information for small animal practitioners. To our knowledge, the present survey is the first reported molecular evidence of feline hemoplasma infections in Romania.

Keywords: Cats, hemotropic mycoplasmas, polymerase chain reaction, Romania

\footnotetext{
* Correspondence: kalman_imre27@yahoo.com

${ }^{6}$ Department of Animal Production and Veterinary Public Health, Faculty of Veterinary Medicine, Banat's University of Agricultural Sciences and Veterinary Medicine "King Michael I of Romania", Timişoara 300645, Romania

Full list of author information is available at the end of the article
}

(C) The Author(s). 2020 Open Access This article is licensed under a Creative Commons Attribution 4.0 International License, which permits use, sharing, adaptation, distribution and reproduction in any medium or format, as long as you give appropriate credit to the original author(s) and the source, provide a link to the Creative Commons licence, and indicate if changes were made. The images or other third party material in this article are included in the article's Creative Commons licence, unless indicated otherwise in a credit line to the material. If material is not included in the article's Creative Commons licence and your intended use is not permitted by statutory regulation or exceeds the permitted use, you will need to obtain permission directly from the copyright holder. To view a copy of this licence, visit http://creativecommons.org/licenses/by/4.0/ The Creative Commons Public Domain Dedication waiver (http://creativecommons.org/publicdomain/zero/1.0/) applies to the data made available in this article, unless otherwise stated in a credit line to the data. 


\section{Background}

The hemotropic mycoplasmas (hemoplasmas) of the genus Mycoplasma within the Mollicutes are recognized as a class of small, wall-less, and uncultivable bacteria that parasitize red blood cells, causing hemolytic anemia in many mammalian species, including cats $[1,2]$.

Results of several molecular investigations conducted at a worldwide level have shown the involvement of three species in the etiology of hemoplasma infections in cats: Mycoplasma haemofelis (Mhf), "Candidatus Mycoplasma haemominutum" (CMhm), and "Candidatus Mycoplasma turicensis" (CMt) (reviewed by [2]). Of these, Mhf has been reported to be the most pathogenic species, being most often associated with clinical disease [3], whereas CMhm and CMt usually result in infections in individuals with concurrent retroviral, neoplastic and immune-mediated disease [1]. In addition, the zoonotic potential of some hemoplasma species has been described. Particularly, "Candidatus M. haemohominis", "Candidatus M. haematoparvum", M. ovis-like, and M. haemofelis have been occasionally related to human infections [4-7].

Hemoplasma infections in cats can vary in severity of symptoms, ranging from complete absence of clinical abnormalities to mild or severe, sometimes fatal acute hemolytic anemia [1]. Currently, the natural route of transmission of hemoplasmas between cats is unknown, but several modes have been suggested, including involvement of arthropod vectors (e.g., fleas, ticks) [8-11], direct transmission via blood transfusion [11], aggressive interactions between cats [12] and transplacental transmission from mother to kittens [13].

Over the last decade, the problem of continuous expansion and emergence of pathogens in novel geographical areas has attracted significant attention from researchers. In this regard, providing new data on the occurrence of unreported pathogens in an endemic area represents a priority for veterinary practitioners and the scientific community. Hemoplasma infections in cats have been molecularly confirmed in several southwestern [14-18], southern [19-21], south-eastern [22, 23], central $[11,24,25]$ and northern [26] countries of Europe. However, to date, no data on the presence of feline hemoplasma infections has been reported in Romania. The present study was undertaken to address this gap by investigating the occurrence of hemoplasmas in cats from western Romania and performing the molecular characterization of the species present.

\section{Results}

Overall, a total of 11 out of 51 domestic cats (21.6\%, 95\% CI 11.8-35.7) tested positive for hemoplasma by PCR amplification of the 16S rRNA gene. Two species were identified: CMhm $(8 / 11 ; 72.7 \%)$ was the dominant species, and Mhf $(3 / 11 ; 27.3 \%)$ was also present in a lower percentage of cats. No co-infections were registered and CMt was not detected in any of the cats. The GAPDH gene was successfully identified in blood samples of all hemoplasma-negative cats. Sequencing of PCR products was successfully performed in all positive samples. Sequence identity analysis showed that the eight CMhm and three Mhf strains derived from the present study were $98.9-100 \%$ and $97.1-100 \%$ homologous to each other, respectively. The sequences showed $>99 \%$ similarity to other GenBank-deposited CMhm (Accession no. KR905451) and Mhf (Accession no. KR905465) sequences from Italy isolated from domestic cats. Detailed distribution of the feline hemotropic mycoplasmas in accordance with the registered epidemiological data are shown in Table 1 . No correlation was found $(p>$ 0.05 ) between hemoplasma infections and the age, gender, breed, presence of ectoparasites, FeLV/FIV positivity status of cats, or the sampling season. However, cats with outdoor access (36.8\%, 95\% CI 17.2-61.4) were found to be more susceptible to hemoplasma infections than those without outdoor access (12.5\%, 95\% CI 4.1$29.9, p=0.049)$. These results suggest that outdoor access could be considered a risk factor $(\mathrm{OR}=4.1,95 \% \mathrm{CI}$ 1.0-16.6) in the acquisition of hemotropic mycoplasmas in cats.

Phylogenetic analysis based on $16 \mathrm{~S}$ rRNA gene sequences showed the Mycoplasma species derived from the current survey and included in the tree construction clustered with those selected from the GenBank database. Thus, our CMhm (accession no. MH223462) and Mhf (accession no. MH223461) isolates branched separately, into one of the two resulting main clades, together with other sequences isolated from cats in Italy (accession no. EU839984) and the United States (accession no. AY069948), respectively (Fig. 1).

Results of the population genetic indices analyses performed separately for each species, as well as for all the Mycoplasma population sequences of $16 \mathrm{~S}$ rRNA gene grouped together are presented in Table 2. The Tajima neutrality test yielded a value of $+2,1692$, and the findings were significant $(P<0.05)$.

\section{Discussion}

To the authors' knowledge, this is the first molecular study documenting the occurrence of hemotropic mycoplasma infections in cats from Romania, expanding the current knowledge of feline hemoplasmas in mainland Europe.

Compared to several other European countries, the hemoplasma prevalence $(21.6 \%)$ was higher than that reported in Spain (12.0\%, [15]; 10.6\%, [18]), Italy (18.9\% [19]; 13.2\%, [21]), Germany (15.6\%, [24]), Denmark (16.4\%, [26]) and Serbia (17.2\%, [23]), similar to that 
Table 1 Distribution of hemoplasma infection in cats according to epidemiological data

\begin{tabular}{|c|c|c|c|c|c|}
\hline \multirow{2}{*}{$\begin{array}{l}\text { Epidemiological } \\
\text { data }\end{array}$} & \multirow{2}{*}{$\begin{array}{l}\text { Total no. of } \\
\text { hemoplasma } \\
\text { infected/tested } \\
\text { cats }(\%)(95 \% \mathrm{Cl})\end{array}$} & \multicolumn{2}{|c|}{ Species identified (number) } & \multirow{2}{*}{$\begin{array}{l}\text { Odds ratios } \\
\text { (OR) }(95 \% \mathrm{Cl})\end{array}$} & \multirow[t]{2}{*}{$p$ value } \\
\hline & & CMhm & Mhf & & \\
\hline \multicolumn{6}{|l|}{ Age } \\
\hline Young ( $\leq 1$-year old $)$ & $2 / 13(15.4)(2.7-46.3)$ & 0 & 2 & $1.7(0.31-9.17)$ & 0.533 \\
\hline Adult (> 1-year old) & 9/38 (23.7) (12.0-40.6) & 8 & 1 & & \\
\hline \multicolumn{6}{|l|}{ Gender } \\
\hline Female & 4/28 (14.3) (4.7-33.6) & 3 & 1 & $2.62(0.7-10.5)$ & 0.171 \\
\hline Male & 7/23 (30.4) (14.1-53.0) & 5 & 2 & & \\
\hline \multicolumn{6}{|l|}{ Breed } \\
\hline European & $7 / 35(20.0)(9.1-37.5)$ & 4 & 3 & $1.33(0.3-5.4)$ & 0.687 \\
\hline Non-European & 4/16 (25.0) (8.3-52.6) & 4 & 0 & & \\
\hline \multicolumn{6}{|l|}{ Sampling season } \\
\hline Warm & 7/31 (22.6) (10.3-41.5) & 6 & 1 & $1.16(0.3-4.6)$ & 0.826 \\
\hline Cold & 4/20 (20) (6.6-44.3) & 2 & 2 & & \\
\hline \multicolumn{6}{|l|}{ Outdoor access } \\
\hline Yes & 7/19 (36.8) (17.2-61.4) & 5 & 2 & $4.1(1.0-16.6)$ & 0.049 \\
\hline No & 4/32 (12.5) (4.1-29.9) & 3 & 1 & & \\
\hline \multicolumn{6}{|c|}{ Presence of ectoparasites } \\
\hline Yes & $3 / 11$ (27.3) (7.3-60.7) & 3 & 0 & $0.66(0.1-3.1)$ & 0.605 \\
\hline No & $8 / 40$ (20.0) (9.6-36.1) & 5 & 3 & & \\
\hline \multicolumn{6}{|l|}{ FeLV status } \\
\hline Positive & 1/5 (20.0) (1.1-70.1) & 0 & 1 & $1.11(0.1-11.1)$ & 0.928 \\
\hline Negative & 10/46 (21.7) (11.5-36.8) & 8 & 2 & & \\
\hline \multicolumn{6}{|l|}{ FIV status } \\
\hline Positive & 2/6 (33.3) (6.0-75.9) & 2 & 0 & $2.00(0.3-12.7)$ & 0.462 \\
\hline Negative & 9/45 (20.0) (10.1-35.1) & 6 & 3 & & \\
\hline Total & $11 / 51(21.6)(11.8-35.7)$ & & & & \\
\hline
\end{tabular}

described in Greece (20.6\%, [22]), but lower than that reported in Portugal (27.1\%, [17]; 43.4\%, [16]) or northern Italy $(33.1 \%$; [20]). It is important to mention that comparing the results of different studies should be undertaken with caution, because differences in study design (e.g. sample size, sampling strategy), epidemiological parameters of the sampled population (e.g. health status of cats, cat's living environment), and the molecular diagnostic techniques used (e.g., conventional PCR, real-time PCR, or a combination of both) in processing the blood samples in different studies could be considered sources of variation for the recorded hemoplasma prevalence. In this regard, it is important to highlight that in our study there was a bias towards the elevation of the infection prevalence, because all the investigated animals were clinically ill at presentation and sampling with suggestive signs for hemoplasma infections.

In accordance with our results, the dominance of CMhm in the screened feline population has been previously confirmed in other molecular surveys [11, 15-26], but others have reported Mhf as the predominant species [14]. It has been hypothesized previously [30] that $\mathrm{CMHm}$ has a more efficient replication and infection capacity but is associated with a lower pathogenic potential compared to the other two species of hemoplasma and often results in asymptomatic carriage status in cats. This can support its dominant occurrence, especially in investigations that report infections in clinically healthy cats. However, in our study considering that all the enrolled animals were clinically ill, without hematological examination, the evaluation of the pathogenicity of the recorded mycoplasma species remains an open question for future investigation.

The carriage of multiple hemoplasma species, as well as the presence of CMt was not detected. Results of other studies have shown that co-infections with different combination of hemoplasmas frequently occur [18, 21, 23], and CMt seems to be the least frequently 


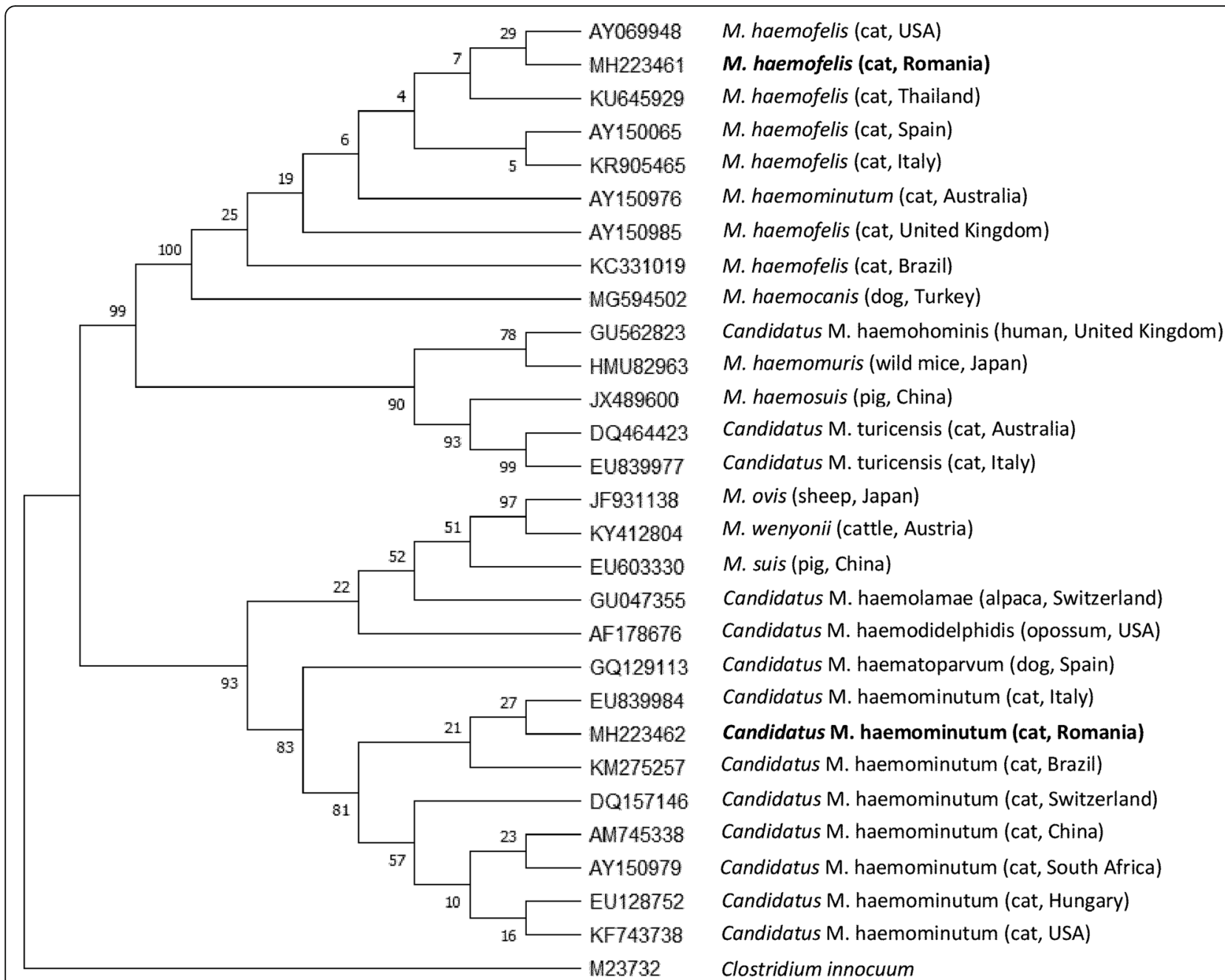

Fig. 1 Phylogenetic tree showing the relationship of the cat origin Mycoplasma sequences derived from the present study (marked with bold) and other Mycoplasma spp. isolated from different hosts in different countries (in brackets), based on analysis of a partial sequence of the 16S rRNA gene. GenBank accession numbers are shown, and Clostridium innocuum (GenBank: M23732) was used as the outgroup. Numbers at branches indicate bootstrap support levels (1,000 replicates). The evolutionary history was inferred by using the Maximum Likelihood method and Tamura-Nei model [27]. The bootstrap consensus tree inferred from 1000 replicates is taken to represent the evolutionary history of the taxa analyzed [28]. Branches corresponding to partitions reproduced in less than $50 \%$ bootstrap replicates are collapsed. The percentage of replicate trees in which the associated taxa clustered together in the bootstrap test (1000 replicates) are shown next to the branches [28]. Initial tree(s) for the heuristic search were obtained automatically by applying Neighbor-Join and BioNJ algorithms to a matrix of pairwise distances estimated using the Tamura-Nei model, and then selecting the topology with superior log likelihood value. This analysis involved 29 nucleotide sequences. Codon positions included were 1st $+2 \mathrm{nd}+3 \mathrm{rd}+$ Noncoding. There was a total of 538 positions in the final dataset. Evolutionary analyses were conducted in MEGA X [29]

Table 2 Genetic indices of the registered cat origin 11 Mycoplasma sequences of 165 rRNA gene

\begin{tabular}{lcclllll}
\hline $\begin{array}{l}\text { Mycoplasma } \\
\text { species }\end{array}$ & $\mathbf{n}$ & S & K & H & Hd \pm S.D. & I S.D. & D \\
\hline CMhm & 8 & 3 & 0.70 & 2 & $0.233 \pm 0.126$ & $0.0014 \pm 0.0007$ & $-0,6544$ \\
Mhf & 3 & 0 & 0.0000 & 1 & 0.0000 & 0.0000 & 0,0000 \\
Total & 11 & 133 & 56.57 & 3 & $0.5520 \pm 0.089$ & $0.1176 \pm 0.0240$ & $+2,1692^{*}$ \\
\hline
\end{tabular}

$n=$ number of sequences examined, $S=$ number of variable sites, $K=$ average number of nucleotide differences, $H=$ number of haplotypes, $H d=$ haplotype diversity, $\pi=$ nucleotide diversity, S.D. standard deviation, $D$ Tajima's $D$ test statistics; statistical significance $={ }^{*} P<0.05$; 
encountered feline hemotropic mycoplasma species, with its prevalence ranging from 0.5 to $6.2 \%[11,15,16,18$, $23]$. In other investigations $[14,22,26]$, in which a limited number of samples were processed as in the case of our study, the lack of the CMt detection was reported. Therefore, further studies, processing a significantly larger number of samples, are still necessary to obtain a more accurate overview and to conclude whether CMt is implicated in Romanian cats' mycoplasma infections.

Outdoor access was the only epidemiological measure that was associated with hemoplasma infection in this study. Other studies have described several factors significantly associated with the presence of hemotropic mycoplasmas in cats, including adult [18] or older [11, $22,24-26,30]$ age, male gender [11, 18, 21, 23-25, 30], non-pedigree breed [23], collection of blood during warm months $[18,19]$, and FeLV/FIV positivity status [15-19, 21, 23-25, 30]. Similar to our finding, the increased likelihood of cats being infected with hemotropic mycoplasmas with outdoor access has been frequently reported in other studies $[11,15,18,23]$. This observation can be sustained by the fact that this lifestyle increases, diversifies and perpetuates the relationships between cats, resulting in the possible transmission of mycoplasma from positive to negative animals, via direct (e.g., fighting or biting) or vector-borne (fleas or ticks) transmission, as have been previously suggested [8-12, $15,21,30]$. However, until now, the scientific demonstration of this hypothesis has remained, until now, unfulfilled.

The structure of the constructed tree, based on $16 \mathrm{~S}$ rRNA gene phylogeny, demonstrated that our isolates were grouped in distinct clades, together with other GenBank-deposited Mycoplasma sequences isolated from cats in different countries from various parts of the world. This phenomenon has been previously observed by Tasker et al. [31]. However, it is important to note that the evolutionary distance analysis indicate the same bootstrap support level between Mhf and M. haemocanis and very similar between CMt and Candidatus M. haematoparvum. The observed unreliable division between these different host-specific mollicutes suggests the use of a more reliable and representative marker in supporting the existence of genetic differences between hemoplasmas infecting cats and dogs [31].

The relatively high values of the majority $(\mathrm{S}, \mathrm{K}, \mathrm{Hd})$ from the registered total population genetic indices (Table 2), together with the significantly positive value of the Tajima neutrality test, are strongly supportive of the great variance in sequences between the recorded two-cat origin Mycoplasma species. In the case of CMhm, the obtained low nucleotide diversity $(\pi)$ value is suggestive of the occurrence of two closely related haplotypes which differ in a small number of nucleotides. Also, the observation of a total high haplotype and relatively low nucleotide diversity within the Mycoplasma population may suggestive for a possible population genetic expansion pattern in the future, as has been previously reported [32].

\section{Conclusions}

The present study provides data on the occurrence of hemoplasma infections as well as the molecular evidence of CMHm and Mhf supported by phylogenetic and population genetic analysis in domestic cats for the first time in Romania. As such, it provides the first indication of the prevalence of hemoplasmas in this previously uninvestigated territory and useful information for small animal practitioners. It implicates the outdoor access lifestyle as a risk factor in the acquisition of disease. The occurrence of feline hemoplasmas in this geographical area, previously thought to be hemoplasma free, opens the opportunity for a larger scale study to be carried out to address some of the limitations of the current survey. In this regard, further studies focusing on the relationship between the hemoplasma species and the resultant hematologic profile, with special emphasis on anemia are recommended.

\section{Methods}

\section{Sample collection and anamnesis}

From April 2017 to February 2019, a total of 51 clientowned cats originating from Timişoara Municipality were presented at the Veterinary Clinics of the Faculty of Veterinary Medicine in Timisoara, Romania, for medical consultation showing one or more suggestive clinical signs (e.g., pallor of the mucous membranes, intermittent pyrexia, weight loss, lethargy, dehydration, or weakness) for feline hemoplasma infections. On the day of presentation, in addition to routine physical examination, all cats were screened for feline leukemia virus (FeLV) and feline immunodeficiency virus (FIV) status using a commercial kit (IDEXX Laboratories, Inc., Westbrook, Maine, USA). In addition, blood was collected from each cat into sterile vacuum tubes with ethylenediaminetetraacetic acid (EDTA) from the antebrachial cephalic vein, to test the presence of hemoplasma DNA. During anamnesis, owners provided information about the cat, including age, gender, breed, outdoor access and any history of previous ectoparasites (i.e., ticks and/or fleas) infestation were collected during anamnesis.

\section{Molecular diagnosis}

As polymerase chain reaction (PCR) assays provide a far more specific and sensitive approach to hemoplasma identification than cytology of blood -smears [2], all biological samples were directly subjected to molecular processing. Genomic DNA was isolated using a PureLink ${ }^{\text {TM }}$ 
genomic DNA mini kit (Invitrogen ${ }^{\mathrm{TM}}$, Carlsbad, CA, USA) according to the manufacturer's instructions. Detection of feline hemotropic mycoplasmas was carried out using conventional PCR based on the amplification of a partial sequence of the $16 \mathrm{~S}$ rRNA gene. The specific forward (5'ACGAAAGTCTGATGGAGCAATA-3') and reverse (5' ACGCCCAATAAATCCGRATAAT-3') primers and cycling parameters were used as previously described by [33]. These primers produce a $193 \mathrm{bp}$ amplified fragment in CMhm and 170 bp in Mhf or CMt. Next, differentiation between Mhf and CMt in PCR-positive samples was carried out using a second PCR protocol with the CMtspecific forward (5'-AGAGGCGAAGGCGAAAACT-3') and reverse (5'-CTACAACGCCGAAACACAAA-3') primers and cycling conditions according to [30, 34]. Subsequently, to obtain a longer Mycoplasma sequence, and for a better molecular assessment of infections in cats, a third conventional PCR was achieved for the previously obtained PCR-positive results, using the universal HBTforward (5'-ATACGGCCCATATTCCTACG-3') and HBT-reverse (5' -TGCTCCACCACTTGTTCA-3') primer set and cycling conditions designed by [14]. The positive PCR reactions produce a $595 \mathrm{bp}$ and $618 \mathrm{bp}$ long amplicons for Mhf and CMhm, respectively. CMhm (reference sequence AF271154) and Mhf (reference sequence AF178677) DNA isolated from blood of naturally infected cats (Diagnostic Laboratories, Langford Vets, University of Bristol, UK), previously confirmed by sequencing, was used as the positive control. The negative control consisted of sterile deionized water. In hemoplasma negative DNA samples, an internal control assay was run targeting the housekeeping feline glyceraldehyde 3-phosphate dehydrogenase (GA3PDH) gene, to ensure the presence of the amplifiable DNA, as well as the absence of PCR inhibitors. The PCRs were performed as described previously [35] using the GAPDH - forward (5'-CCTTCATTGA CCTCAACTACAT-3') and GAPDH - reverse (5'-CCAA AGTTGTCATGGATGACC-3') specific primer set.

All PCR products were visualized on Midori Green (Nippon Genetics ${ }^{\circ}$; Europe, Gmbh) stained 1.8\% agarose gel.

The PCR amplified 16S rRNA gene amplicons showing positive results were purified (Isolate II PCR and Gel Kit, Bioline $^{\mathrm{TM}}$ ) and bidirectionally sequenced (performed by Macrogen $^{\mathrm{Tm}}$ Europe, Amsterdam, the Netherlands). The resulting sequences were analyzed for their homology using the Clustal Omega (available online: https://www. ebi.ac.uk/Tools/msa/clustalo/) multiple sequence alignment program and subjected to BLAST (Basic Local Alignment Search Tool) analysis to compare them to those available in the GenBank ${ }^{\mathrm{Tm}}$ dataset. All the obtained sequences were deposited into GenBank ${ }^{\mathrm{Tm}}$ as follows: Mhf: MH223461 and MT926037 - MT926038; and CMhm: MH223462 and MT926039 - MT926045.

\section{Phylogenetic and population genetic analysis}

Phylogenetic analysis was performed with MEGAX (ver. 10.1) software [29]. The evolution of two representative DNA sequences from the present study and those available from the GenBank database were measured by the Tamura-Nei model [27]. The tree was constructed using the maximum likelihood method with 1000 bootstrap replicates. The characterization of the bacterial strain population diversity indices, including the nucleotide diversity $(\pi)$, haplotype diversity $(\mathrm{Hd})$, number of variable sites (S), and nucleotide differences $(\mathrm{K})$, was carried out using the DnaSP (ver. 6.12) software [36]. In addition, the Tajima neutrality test was also computed [37].

\section{Statistical analysis}

Statistical analyses were performed using SPSS 21.0 software. The possible association between the hemoplasma infection status of cats and the recorded epidemiological parameters was assessed with the nonparametric Pearson's chi - squared $\left(\chi^{2}\right)$ test. Differences were established as statistically significant when $p$ value $\leq 0.05$. Also, the risk factors were evaluated through calculation of odds ratios (ORs) with 95\% confidence intervals (CIs) by including each variable in the binary Logit model of the multivariate regression analysis.

\section{Abbreviations}

Mhf: Mycoplasma haemofelis; CMhm: Candidatus Mycoplasma haemominutum; CMt: Candidatus Mycoplasma turicensis; FeLV: feline leukemia virus; FIV: feline immunodeficiency virus; DNA: deoxyribonucleic acid; EDTA: ethylenediaminetetraacetic acid; PCR: polymerase chain reaction; RNA: ribonucleic acid; BLAST: Basic Local Alignment Search Tool; OR: odds ratio; Cl: confidence interval

\section{Acknowledgements \\ The authors appreciate very much the final year veterinary students' cooperation and assistance during investigations.}

\section{Authors' contributions}

$\mathrm{MI}$ and TS performed laboratory work and participated in data analysis. CV, performed the sampling and clinical evaluation of cats. GD, SM and VH

helped with the study design and implementation, and data interpretation. $J P$, performed the phylogenetic and population genetic analysis. PJPL contributed to the Discussion and participated in the writing the manuscript. $\mathrm{Kl}$ conceived and designed the study, coordinated the research team, and drafted the manuscript. All authors read and approved the final manuscript.

\section{Funding}

The publication of this research paper is supported by the project "Ensuring excellence in the activity of RDI within USAMVBT" code 35PFE, submitted in the competition Program 1 - Development of the national system of research - development, Subprogram 1.2 - Institutional performance, Institutional development projects - Development projects of excellence in RDI". The funding body had no role in the design of the study and collection, analysis, and interpretation of data and in writing the manuscript.

\section{Availability of data and materials}

The sequences (accession numbers MH223461, MT926037 - MT926038, MH223462, and MT926039 - MT926045) used to support the findings of this study have been deposited in the GenBank repository. The datasets generated and analyzed during the current study are included within the article. 


\section{Ethics approval and consent to participate}

The research protocol was reviewed and approved by the Research Ethics Committee of BUASVM "King Michail I of Romania", Timișoara, Romania, and registration No. $128-06.12 .2018$. Written consent was obtained from cat owners for blood samples to be used in research/publication.

\section{Consent for publication}

Not applicable.

\section{Competing interests}

The authors declare that they have no competing interests.

\section{Author details}

'Department of Parasitology and Parasitic Diseases, Faculty of Veterinary Medicine, Banat's University of Agricultural Sciences and Veterinary Medicine "King Michael I of Romania", Timişoara 300645, Romania. ${ }^{2}$ Department of Internal Medicine, Faculty of Veterinary Medicine, Banat's University of Agricultural Sciences and Veterinary Medicine Timişoara, Calea Aradului no. 119, 300645 Timişoara, Romania. ${ }^{3}$ Department of Infectious Diseases and Preventive Medicine, Faculty of Veterinary Medicine, Banat's University of Agricultural Sciences and Veterinary Medicine "King Michael I of Romania", Timişoara, Romania. ${ }^{4}$ Department of Environmental Health, National Public Health Center, 1097 Budapest, Hungary. ${ }^{5}$ Langford Vets, University of Bristol, Langford, UK. ${ }^{6}$ Department of Animal Production and Veterinary Public Health, Faculty of Veterinary Medicine, Banat's University of Agricultural Sciences and Veterinary Medicine "King Michael I of Romania", Timişoara 300645, Romania.

\section{Received: 5 March 2020 Accepted: 15 October 2020}

Published online: 22 October 2020

\section{References}

1. Rani N, Tomar P, Kapoor PK, Singh Y. A Review on Emerging Zoonotic Mycoplasma. Int J Pure App Biosci. 2018;6:784-90.

2. Tasker S, Hofmann-Lehmann R, Belák S. Haemoplasmosis in cats: European guidelines from the ABCD on prevention and management. J Feline Med Surg. 2018;20:256-61.

3. Tasker S. Haemotropic mycoplasmas: what's their real significance in cats? J Feline Med Surg. 2010;12:369-81.

4. Steer JA, Tasker S, Barker EN, Jensen J, Mitchell J, Stocki T, Chalker VJ, Hamon M. A novel hemotropic Mycoplasma (Hemoplasma) in a patient with hemolytic anemia and pyrexia. Clin Infect Dis. 2011;53:147-51.

5. Hattori N, Kuroda M, Katano H, Takuma T, Ito T, Arai N, Yanai R, Sekizuka T, Ishii S, Miura Y, Tokunaga T, Watanabe H, Nomura N, Eguchi J, Hasegawa H, Nakamaki T, Wakita T, Niki Y. Candidatus Mycoplasma haemohominis in human, Japan. Emerg Infect Dis. 2020;26:11-9.

6. Maggi RG, Compton SM, Trull CL, Mascarelli PE, Mozayeni BR, Breitschwerdt EB. Infection wit Hemotropic Mycoplasma species in patients with or without extensive arthropod or animal contact. J Clin Microbiol. 2013;51: $3237-41$.

7. Dos Santos AP, Dos Santos RP, Biondo AW, Dora JM, Goldani LZ, de Oliveira ST, de Sá Guimařaes AM, Timenetsky J, de Morais A, González FHD, Messick JB. Hemoplasma infection in HIV-positive patient, Brazil. Emerg Infect Dis. 2008;14:1922-24.

8. Shaw SE, Kenny MJ, Tasker S, Birtles RJ. Pathogen carriage by the cat flea Ctenocephalides felis (Bouché) in the United Kingdom. Vet Microbiol. 2004; 102:183-88.

9. Taroura S, Shimada Y, Sakata Y, Miyama T, Hiraoka H, Watanabe M,et al. Detection of DNA of "Candidatus Mycoplasma haemominutum" and Spiroplasma sp. in unfed ticks collected from vegetation in Japan. J Vet Med Sci. 2005;67:1277-9.

10. Woods JE, Brewer MM, Hawley JR, Wisnewski N, Lappin MR. Evaluation of experimental transmission of Candidatus Mycoplasma haemominutum and Mycoplasma haemofelis by Ctenocephalides felis to cats. Am J Vet Res. 2005; 66:1008-12.

11. Willi B, Boretti FS, Baumgartner C, Tasker S, Wenger B, Cattori V, et al. Prevalence, risk factor analysis, and follow-up of infections caused by three feline hemoplasma species in cats in Switzerland. J Clin Microbiol. 2006;44: 961-69.
12. Museux K, Boretti FS, Willi B, Riond B, Hoelzle K, Hoelzle LE, et al. In vivo transmission studies of 'Candidatus Mycoplasma turicensis' in the domestic cat. Vet Res. 2009:40:45.

13. Fisher EW, Toth S, Collier WO. Anaemia in a litter of Siamese kittens. J Small Anim Pract. 1983;24:214-19.

14. Criado-Fornelio A, Martinez-Marcos A, Buling-Saraña A. Barba-CarreteroJC. Presence of Mycoplasma haemofelis, Mycoplasma haemominutum and piroplasmids in cats from southern Europe: a molecular study. Vet Microbiol. 2003;93:307-17.

15. Roura X, Peters IR, Altet L, Tabar MD, Barker EN, Planellas M,et al. Prevalence of hemotropic mycoplasmas in healthy and unhealthy cats and dogs in Spain. J Vet Diagn Invest. 2010;22:270-74.

16. Martínez-Díaz VL, Silvestre-Ferreira AC, Vilhena H, Pastor J, Francino O, Altet L. Prevalence and co-infection of haemotropic mycoplasmas in Portuguese cats by real-time polymerase chain reaction. J Feline Med Surg. 2013;15:879-85.

17. Duarte A, Marques V, Correia JHD, Neto I, Bráz BS, Rodrigues C, et al. Molecular detection of haemotropic Mycoplasma species in urban and rural cats from Portugal. J Feline Med Surg. 2015;17:516-22.

18. Díaz-Regañón D, Villaescusa A, Ayllón T, Franco FR, Sancho MG, Agulla B, Sainz Á. Epidemiological study of hemotropic mycoplasmas (hemoplasmas) in cats from central Spain. Parasit Vectors. 2018;11:140.

19. Gentilini F, Novacco M, Turba ME, Willi B, Bacci ML, Hofmann-Lehmann R. Use of combined conventional and real-time PCR to determine the epidemiology of feline haemoplasma infections in northern Italy. J Feline Med Surg. 2009;11:277-85.

20. Spada E, Proverbio D, Galluzzo P, Della Pepa A, Bagnagatti De Giorgi G, Perego R, Ferro E. Prevalence of haemoplasma infections in stray cats in northern Italy. Int Sch Res Not. 2014;2014(298352):8. https://doi.org/10.1155/ 2014/298352.

21. Ravagnan S, Carli E, Piseddu E, Da Rold G, Porcellato E, Zanardello C, et al. Prevalence and molecular characterization of canine and feline hemotropic mycoplasmas (hemoplasmas) in northern Italy. Parasit Vectors. 2017;10:132.

22. Maher IE, Tasker S, Polizopoulou Z, Dasopoulou A, Egan K, Helps CR, Papasouliotis K. Polymerase chain reaction survey of feline haemoplasma infections in Greece. J Feline Med Surg. 2010;12:601-5.

23. Sarvani E, Tasker S, Kovácević FM, Francuski AJ, Andric N, Anquino L, et al. Prevalence and risk factor analysis for feline haemoplasmas in cats from Northern Serbia, with molecular subtyping of feline immunodeficiency virus. J Feline Med Surg. 2018;4:1-10.

24. Just F, Pfister K. Detection frequency of haemoplasma infections of the domestic cat in Germany. Berl Munch Tierarztl Wochenschr. 2007;120:197-201.

25. Bauer N, Balzer HJ, Thüre S, Moritz A. Prevalence of feline haemotropic mycoplasmas in convenience samples of cats in Germany. J Feline Med Surg. 2008;10:252-58.

26. Rosenqvist MB, Meilstrup AKH, Larsen J, Olsen JE, Jensen AL, Thomsen LE. Prevalence of feline haemoplasma in cats in Denmark. Acta Vet Scand. 2016; $58: 78$.

27. Tamura K, Nei M. Estimation of the number of nucleotide substitutions in the control region of mithocondrial DNA in humans and chimpanzees. Mol Biol Evol. 1993;10:512-26.

28. Felsenstein J. Confidence limits on phylogenies: An approach using the bootstrap. Evolution. 1985;39:783-791.

29. Kumar S, Stecher G, Li M, Knyaz C, Tamura K. MEGA X: Molecular Evolutionary Genetics Analysis across computing platforms. Mol Biol Evol. 2018;35:1547-9.

30. Tanahara M, Miyamoto S, Nishio T, Yoshii Y, Sakuma M, Sakata Y, et al. An epidemiological survey of feline hemoplasma infection in Japan. J Vet Med Sci. 2010;72:1575-81.

31. Tasker S, Helps CR, Day MJ, Harbour DA, Shaw SE, Harrus S, Baneth G, Lobetti RG, Malik R, Beaufils JP, Belford CR, Gruffydd-Jones TJ. Phylogenetic analysis of Hemoplasma species: an international study. J Clin Microbiol. 2003;41:3877-80.

32. Avise JC. Phylogeography: the history and formation of species. Cambridge: Harvard University Press. 2000;447.

33. Jensen WA, Lappin MR, Kamkar S, Reagan WJ. Use of a polymerase chain reaction assay to detect and differentiate two strains of Haemobartonella felis in naturally infected cats. Am J Vet Res. 2001;62:604-8.

34. Peters IR, Helps CR, Willi B, Hofmann-Lehmann R, Tasker $\mathrm{S}$. The prevalence of three species of feline haemoplasmas in samples submitted to a diagnostics service as determined by three novel real-time duplex PCR assays. Vet Microbiol. 2008;126:142-50. 
35. Birkenheuer AJ, Levy MG, Breitschwerdt EB. Development and evaluation of a seminested PCR for detection and differentiation of Babesia gibsoni (Asian genotype) and B. canis DNA in canine blood samples. J Clin Microbiol. 2003; 41:4172-7.

36. Rozas J, Sánchez-DelBarrio JC, Messeguer X, Rozas R, DnaSP. DNA polymorphism analyses by the coalescent and other methods. Bioinformatics. 2003;19:2496-7.

37. Tajima F. Statistical method for testing the neutral mutation hypothesis by DNA polymorphism. Genetics. 1989;123:585-95.

\section{Publisher's Note}

Springer Nature remains neutral with regard to jurisdictional claims in published maps and institutional affiliations.

Ready to submit your research? Choose BMC and benefit from:

- fast, convenient online submission

- thorough peer review by experienced researchers in your field

- rapid publication on acceptance

- support for research data, including large and complex data types

- gold Open Access which fosters wider collaboration and increased citations

- maximum visibility for your research: over $100 \mathrm{M}$ website views per year

At $\mathrm{BMC}$, research is always in progress.

Learn more biomedcentral.com/submissions 